\title{
PNEUMONECTOMY IN A CASE OF FISTULA BETWEEN THE MIDDLE LOBE BRONCHUS AND MIDDLE LOBE ARTERY BY
}

\author{
FRANCO SOAVE* \\ From the Surgical Clinic of Sabbatsberg Hospital, Stockholm
}

This case, of a patient treated by pneumonectomy for a fistula between the right middle lobe bronchus and the artery to the right middle lobe, is of interest as no other appears to have been described in the literature.

\section{CASE REPORT}

The patient, a 48-year-old man, was admitted to Professor Crafoord's clinic on December 27, 1947. His mother had died of a cerebral haemorrhage, and one of his brothers had died at 20 years of age of pulmonary tuberculosis. Two of his sisters suffered from cancer and had been treated elsewhere. The patient was healthy as a child. At the age of 20 he began work as a turner. He was married and had two children who were physically and mentally sound.

When he was 24 years old the patient recalled that he had had small haemoptyses. In 1930, when 31 years old, without pulmonary symptoms in the meantime, he had a violent haemoptysis of about $1,500 \mathrm{ml}$. The patient subsequently continued his work in good physical condition for about 12 years and underwent regular medical examinations. These revealed no pulmonary disease, and failed to show any reason for the haemoptyses.

During 1942 the patient had no less than 12 slight haemoptyses. In 1943 bronchoscopy was carried out, and the findings were normal for the larynx, the trachea, the carina, and the left bronchial tree. On the right side there were signs of increasing subacute inflammation of the mucous membrane down to the middle lobe bronchus, whose lumen showed marked oedema and a certain degree of stenosis. A large clot of blood had formed in its lumen. Biopsy of the mucous membrane at the point of the greatest constriction caused a fairly profuse haemorrhage.

The microscopic report indicated the presence of chronic tuberculosis. There were no signs of a neoplastic process.

During the following month repeated examinations, both clinical and radiological, gave negative results. A month later renewed control bronchoscopy was performed with exactly the same findings as before.

The inflamed mucous membrane was treated with lactic acid. The clots in the middle lobe bronchus were removed by suction. A probe passed easily, and no definite stenosis of the middle lobe bronchus could be established. This examination also failed to confirm the histological diagnosis, although chronic tuberculosis was considered to be the most probable cause.

For nearly two years the patient was well and under strict medical control, and in 1945 radiographic examination showed a normal left side, but signs of partial

* Dr. Soave is a member of the Department of Surgery, Genoa University. He recorded his case during a period spent at the Sabbatsberg Hospital, Stockholm. 
atelectasis were present in the posterior part of the middle lobe. At bronchoscopy, after hospitalization for about one month on the grounds of the radiological findings, the mucous membrane was seen to be considerably more inflamed than in the previous examinations and the opening of the middle bronchus to be almost totally blocked.

Microscopic examination of the mucous membrane from the site of greatest infiltration confirmed to some extent the suspicion of bronchial tuberculosis and once again excluded the possibility of a tumour.

From 1945 to November, 1947, the patient had some small haemoptyses without seeking medical advice. On November 26 he was admitted to hospital for a large haemoptysis of about $600 \mathrm{ml}$. During his stay in hospital he had frequent smaller haemoptyses and was therefore transferred to Sabbatsberg Hospital, under the care of Professor C. Crafoord, on December 27, 1947.

Clinical examination revealed that the patient was physically well developed, slightly undernourished, had no dyspnoea, no cyanosis, no clubbing of the fingers or toes. Nothing abnormal was found on cardiac examination. An electrocardio-

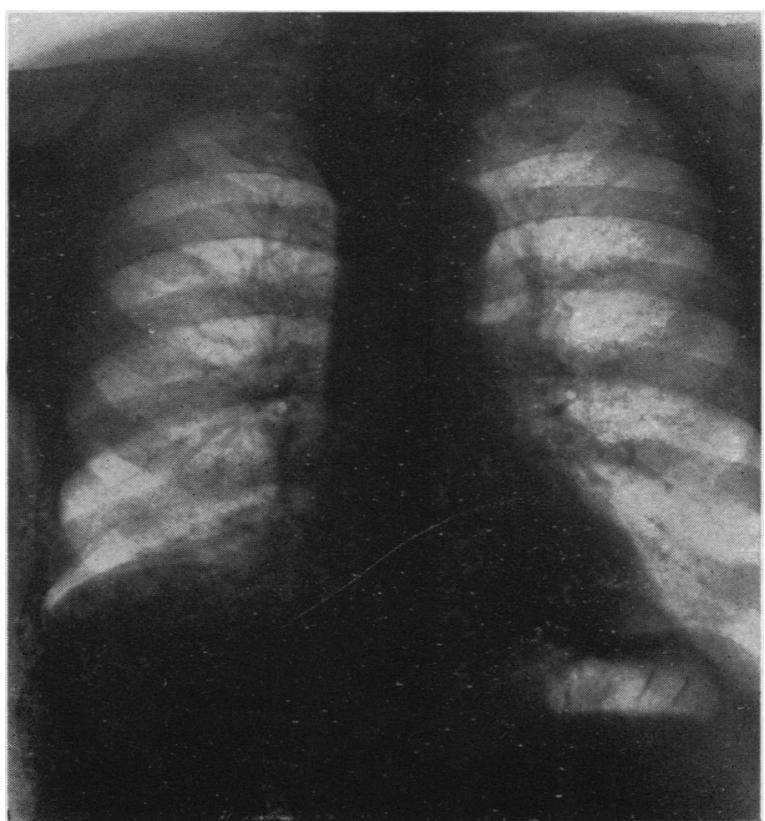

Fig. 1.-A.P. view. A well-defined shadow which has the appearance of a parenchymatous thickening is present in the hilar zone (right side).

gram was normal. There was no clinical sign of disease in the thorax, and the abdomen was normal. There was no cyanosis, and no oedema in the limbs.

Blood pressure was consistently $120 / 75 \mathrm{~mm}$. $\mathrm{Hg}$.

The haemoglobin count varied between 58 and $79 \%$, and the red blood count between 3.2 and 4.5 millions per $\mathrm{mm}^{3}$ The sedimentation rate was $35 \mathrm{~mm}$./ hour.

The hepatic and renal functions were normal.

The Wassermann reaction was negative.

Vital capacity was 2,620 (estimated to 3,385 ).

The sputum was negative.

Radiological examination of the thorax showed that the left side was normal, but on the right side the diaphragm was slightly elevated and there was some decrease in mobility. A well-defined shadow which had the appearance of a parenchymatous thickening was present in the hilar zone in the antero-posterior view (Fig. 1). In the lateral projection this thickening was evidently due to a partial atelectasis of the middle lobe (Fig. 2).

Whilst the anaesthetic was being administered before bronchoscopy on December 30,1947 , the patient had a violent haemoptysis of more than one litre with signs of serious suffocation. The bronchoscope was quickly inserted into the trachea and several big clots and blood were sucked up, thereby freeing the windpipe. The endo- 
scopic examination confirmed the presence of stenosis in the middle lobe bronchus, down the lumen of which it was possible to pass only a very thin probe. It was not possible to localize the origin of the haemorrhage because it had stopped spontaneously when the blood pressure fell and the patient became unconscious. The patient remained in hospital and was prepared for thoracotomy and lung resection.

Professor C. Crafoord decided to perform a thoracotomy with removal of the sixth rib on January 22, 1948. As soon as the pleural cavity had been opened the right main bronchus was dissected and clamped in order to prevent bleeding into

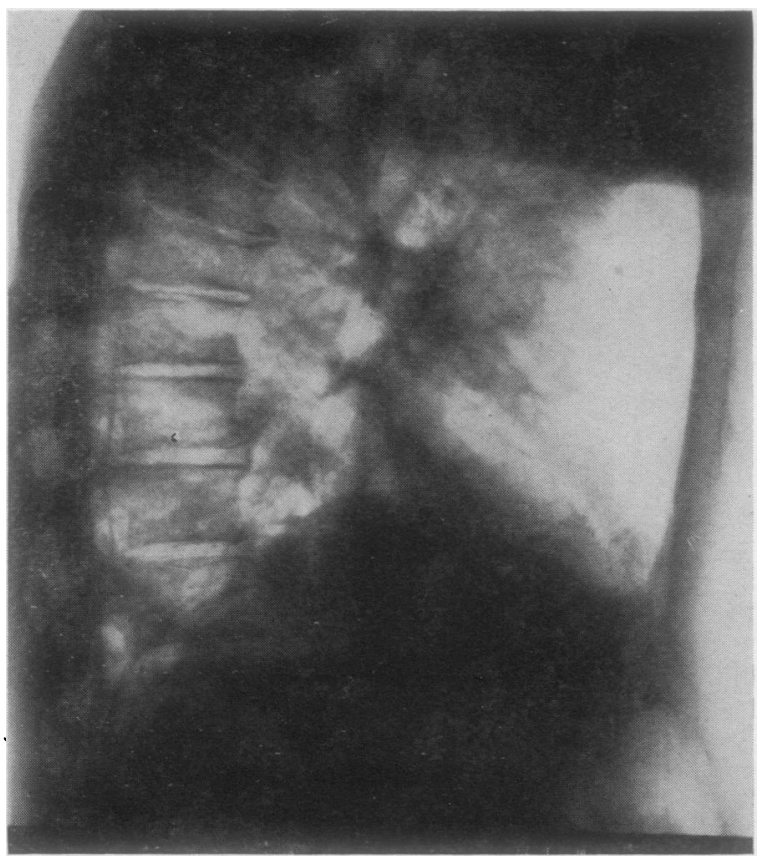

Fig. 2.-L.L. view. Partial atelectasis of the middle lobe. the left lung should haemorrhage occur. The middle lobe was atelectatic. The upper and lower lobes were air-containing. It proved impossible to carry out lobectomy of the middle lobe on account of an extensive inflammatory process in the hilus which prevented anatomical dissection of the vessels and bronchial branch to the middle lobe. A pneumonectomy had to be done. The main bronchus was sectioned and treated with invagination according to Crafoord's technique. The hilar area was closed with continuous suturing of the pleura. The operation was concluded by primary closure of the thoracic wound with continuous suction drainage. The post-operative period was without incident, and the patient was discharged from hospital on February 21, 1948, and has remained well since then.
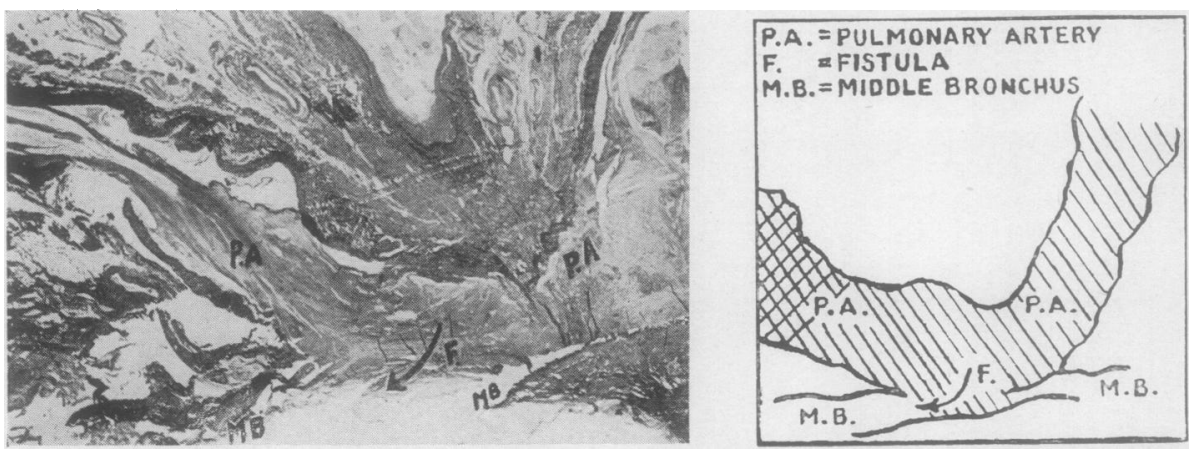

Fig. 3.-Section on microscopic examination. The pulmonary artery is filled with clotted blood. P.A. $=$ Pulmonary artery to the middle lobe; F. $=$ Fistula ; M.B. $=$ Middle lobe bronchus. 
Macroscopic examination showed that the bronchus between the right upper and the right middle lobe bronchus was considerably stenotic. Its mucous membrane was shiny, thin, and pale. In the distal part of the angle formed by the main bronchus and the middle bronchus there was a small round hole with a diameter of $4 \mathrm{~mm}$. within the middle lobe bronchus and in direct communication with the lumen of the pulmonary artery. This opening, which closely soldered together the two elements of the lung peduncle, had smooth and rounded edges and was filled with an elastic, thrombus-like mass.

On microscopic examination of the zone surrounding the fistula an intense chronic inflammation was found. The bronchus on the edges of the blocked zone was wrapped in a strongly sclerotic connective tissue, which in some places was distinctly pigmented, anthracotic, and hyaline. Round the fistula were the remains of a lymph node which was also strongly anthracotic but with no signs of calcification. The elastic tissue of the artery had completely disappeared at the edges of the perforation, which were infiltrated with unspecific chronic inflammatory cells and covered with thrombotic masses (Fig. 3).

\section{Discussion}

It was not possible before the operation to discover the cause of the repeated haemoptyses. Although there was a chronic inflammation of the mucous membrane, there were no grounds on which an exact diagnosis could be based. It was only radiologically that the suspicions of a tumour were avowed but never confirmed. The condition of the mucous membrane, with no signs of granulations or ulcerations during the whole period, could have implied a tuberculous process in the peribronchial tissue or lymph nodes resulting in bronchial obstruction. A perforation between a bronchus and a large blood vessel produced by such a process was the most likely explanation of the symptoms even if this could not be verified before operation. The most probable course of events was the formation of a chronic abscess in a lymph node with gradual erosion of both bronchial and arterial walls in the vicinity of the lymph node, resulting in a communication between the two.

This case should be borne in mind when seeking an explanation for multiple haemoptyses occurring over a long period of time when no other pathological condition can be demonstrated to explain the bleeding.

I take this opportunity of expressing my sincere thanks to Professor Crafoord for his permission to publish this case. 